\title{
MAXIMA OF SUMS OF HEAVY-TAILED RANDOM VARIABLES
}

BY

K.W. $\mathrm{NG}^{1}$, Q.H. TANG ${ }^{2}$, H. YANG

\begin{abstract}
In this paper, we investigate asymptotic properties of the tail probabilities of the maxima of partial sums of independent random variables. For some large classes of heavy-tailed distributions, we show that the tail probabilities of the maxima of the partial sums asymptotically equal to the sum of the tail probabilities of the individual random variables. Then we partially extend the result to the case of random sums. Applications to some commonly used risk processes are proposed. All heavy-tailed distributions involved in this paper are supposed on the whole real line.
\end{abstract}

\section{KEYWORDS}

Asymptotics, Heavy tails, Insurance risk model, Partial sums, Random sums, Subexponential distribution, Tail probabilities.

\section{INTRODUCTION}

Since the classical work of Lévy's formula

$$
\mathbb{P}\left(\sup _{0 \leq t \leq 1} B(t)>x\right)=2 \mathbb{P}(B(1)>x),
$$

where $B(t)$ is the Brownian motion satisfying $B(0)=0$, the question as how to establish relations of the type

$$
\mathbb{P}\left(\sup _{0 \leq t \leq 1} W(t)>x\right) \sim c \mathbb{P}(W(1)>x)
$$

for certain stochastic process $W(t)$ has been in the focus of many researchers, where $c>0$ is a constant. In a special case where $W(t)$, with $W(0)=0$, is a

1 Department of Statistics and Actuarial Science, University of Hong Kong.

2 University of Amsterdam, Department of Quantitative Economics.

ASTIN BULLETIN, Vol. 32, No. 1, 2002, pp. 43-55 
continuous time process with independent and stationary increments, relations like (1.1) have been studied by many authors. Berman (1986) proved (1.1) for $c=1$ under the assumptions that $W(t)$ has symmetric increments and that the tail of its Lévy's spectral measure is of regular variation. Some related papers are Willekens (1987), Braverman \& Samorodnitsky (1995). Most recently, Braverman (2000) further considered the tail asymptotics for the suprema of Lévy process $W(t)$ with light-tailed spectral Lévy measure. After introducing the notation of the right light-tailed distributions, he obtained (1.1) for the compound Poisson process

$$
W(t)=\sum_{k=1}^{N(t)} X_{k}-b t, \quad t \geq 0,
$$

where, $b>0$ is a constant, and $\left\{X_{k}, k \geq 1\right\}$ is a sequence of i.i.d. and lighttailed random variables, independent of the homogeneous Poisson process $N(t)$. See also Braverman (1999). All the works above assume that the process is infinitely divisible.

Now one question naturally arises: does (1.1) holds if $W(t)$ is not an infinitely divisible process? The works cited above give no clue to this question. In this paper we shall establish a result (in Theorem 2.3 below) that is more general than giving a positive answer. From Theorem 2.3 with $\tau$ replaced by a counting process, we can easily obtain asymptotics like (1.1) for some commonly used processes in insurance risk models; see also Example 3.2 below. The methodology we used in this paper is different from those in the references above.

\section{MAIN RESUlts}

Throughout this paper, $\left\{X_{k}, k \geq 1\right\}$ denotes a sequence of independent random variables (r.v.'s), each $X_{k}$ has a distribution function (d.f.), $F_{k}(x)=1-\overline{F_{k}}(x)$ $=\mathbb{P}\left(X_{k} \leq x\right), k \geq 1$. We denote by $S_{n}$ the $n$th partial sum of the sequence $\left\{X_{k}\right.$, $k \geq 1\}$. In the sequel, each $F$, or $F_{k}$, is always assumed to satisfy that $\bar{F}(x)>0$ for all $x$. All limit relationships, unless otherwise stated, are for $x \rightarrow \infty$. For two positive infinitesimals $A(x)$ and $B(x)$, we write $A(x) \leqslant B(x)$ if limsup $A(x) /$ $B(x) \leq 1$, and define the reverse relation $A(x) \geq B(x)$ in the natural way. We write $A(x) \approx B(x)$ if

$$
0<\liminf A(x) / B(x) \leq \lim \sup A(x) / B(x)<\infty .
$$

We further write $A(x) \sim B(x)$, as usual, if both the limits above equal to 1 .

Like many recent researchers in the fields of applied probability and risk theory, we restrict our interest to the case of heavy-tailed risks. A r.v. $X$ (or its d.f. $F$ ) is said to be heavy-tailed on right-hand if $\mathbb{E} e^{r X}=\infty$ for any $r>0$. The most important heavy-tailed subclass is the subexponential class (denoted as $\mathcal{S}$ ). By definition, a d.f. $F$ supported on $[0, \infty)$ belongs to $S$ iff

$$
\lim _{x \rightarrow \infty} \overline{F^{* n}}(x) / \bar{F}(x)=n
$$


for all $n \geq 2$ (or equivalently for some $n \geq 2$ ), where $F^{*}$ denotes the $n$-fold convolution of $F$. That is, for a sequence of i.i.d. r.v.'s $\left\{X_{k}, k \geq 1\right\}$ with a common d.f. $F \in S$, it holds that, for each $n \geq 2$,

$$
\mathbb{P}\left(S_{n}>x\right) \sim \mathbb{P}\left(\max _{1 \leq k \leq n} X_{k}>x\right) .
$$

More generally, a r.v. $X$ with a d.f. supported on $(-\infty, \infty)$ belongs to $S$ if $X^{+}=$ $\max \{0, X\}$ belongs to $S$; see Willekens (1986). There are two other heavytailed subclasses, class $\mathcal{L}$ of long-tailed d.f.'s and class $\mathcal{D}$ of d.f.'s with dominatedly varying tails, which are closely related to class $\mathcal{S}$.. A d.f. $F$ supported on $(-\infty, \infty)$ belongs to class $\mathcal{L}$ iff

$$
\lim _{x \rightarrow \infty} \bar{F}(x+y) / \bar{F}(x)=1
$$

for any $y>0$ (or equivalently for some $y>0$ ); $F$ belongs to class $\mathcal{D}$ iff

$$
\limsup _{x \rightarrow \infty} \bar{F}(x y) / \bar{F}(x)<\infty
$$

for any $0<y<1$ (or equivalently for some $0<y<1$ ). It is well-known that

$$
\mathcal{D} \cap \mathcal{L} \subset \mathcal{S} \subset \mathcal{L}
$$

For details of heavy-tailed subclasses and their applications to insurance and finance, the readers are referred to Embrechts et al. (1997) and Goldie \& Klüppelberg (1998).

In this paper we address the asymptotic properties of the tail probabilities of the maxima of the first $n$ partial sums, $\max _{1 \leq k \leq n} S_{k}$, where $n$ is either a constant or random. Now we state the main results of this paper as follows.

Theorem 2.1. Suppose that the d.f. $F_{k} \in \mathcal{L}$ for $k \geq 1$. Then we have that, for each $n \in \mathcal{N}$.

$$
\mathbb{P}\left(\max _{1 \leq k \leq n} S_{k}>x\right) \sim \mathbb{P}\left(S_{n}>x\right) .
$$

If we restrict ourselves to the subexponential case, from Theorem 2.1 we can obtain

Theorem 2.2. Suppose that the d.f. $\overline{F_{k}}(x)-c_{k} \bar{F}(x)$ for $k \geq 1$, where $\bar{F}$ is the tail of some subexponential d.f. $F$, and $c_{k}, k \geq 1$, are some non-negative constants such that $C(n)=\sum_{k=1}^{n} c_{k}>0$. Then we have that, for each $n \in \mathfrak{N}$,

$$
\mathbb{P}\left(\max _{1 \leq k \leq n} S_{k}>x\right) \sim \mathbb{P}\left(S_{n}>x\right) \sim \mathbb{P}\left(\max _{1 \leq k \leq n} X_{k}>x\right) \sim C(n) \bar{F}(x) .
$$

We remark that, the conclusion $\mathbb{P}\left(\max _{1 \leq k \leq n} S_{k}>x\right) \sim C(n) \bar{F}(x)$ in (2.6) partially coincides with Theorem 1 in Sgibnev (1996). In the latter the author used 
sophisticated analytical arguments of the Pollaczek-Spitzer identity to derive an asymptotic formula similar to (2.6) but only for the i.i.d. case. In this paper we shall provide an elementary proof of (2.6). We suggest the readers to compare our (2.6) with the definition of (2.2).

We continue to state the main results of the paper. In the following theorem we partially extend the asymptotic formula (2.6) to the case of random sums.

Theorem 2.3. Suppose that $\left\{X_{k}, k \geq 1\right\}$ is a sequence of i.i.d. r.v.'s with a common d.f. $F \in \mathcal{L} \cap \mathcal{D}$ and a finite expectation. Let $\tau$ be a non-negative and integervalued r.v., independent of the sequence $\left\{X_{k}, k \geq 1\right\}$ and satisfying that $\mathbb{P}(\tau>x)=$ o $(\bar{F}(x))$. Then,

$$
\mathbb{P}\left(\max _{1 \leq k \leq n} S_{k}>x\right) \sim \mathbb{P}\left(S_{\tau}>x\right) \sim \mathbb{E} \tau \bar{F}(x) .
$$

Note that the conditions in Theorem 2.3 imply the existence of $\mathbb{E} \tau$. It is wellknown that the intersection $\mathcal{L} \cap \mathcal{D}$ is a very large subexponential subclass. It contains many useful subclasses of heavy-tailed distributions. See Bingham et al. (1987) and Embrechts et al. (1997) for details. We also remark that, the second asymptotic relationship in (2.7) describes the tail asymptotic behavior of the compound sum

$$
\mathbb{P}\left(S_{\tau} \leq x\right)=\sum_{n=0}^{\infty} \mathbb{P}(\tau=n) F^{* n}(x) .
$$

For more general discussion about the tail asymptotics of the compound distributions, please refer to Chover et al. (1973), Embrechts et al. (1979), Embrechts \& Goldie (1982) and Cline (1987).

\section{Applications to Risk Processes}

In this section we show some applications of our results to some commonly used risk processes. All of the following examples are complementary to recent research on the tail behavior of the maxima/suprema of processes in discrete/ continuous time.

Example 3.1. We now consider a discrete time insurance risk model. Upper bounds for the ultimate ruin probability under this model were discussed in Yang (1999). Suppose within the $k$ th time period, the total claim size is $Z_{k}$ and the total premium income is $Y_{k}$, and the claim is paid at the end of the time period while the premium is paid at the beginning. We assume $\left\{\left(Z_{k}, Y_{k}\right)\right.$, $k \geq 1\}$ constitutes a sequence of independent, but not necessarily identically distributed, random vectors. Let $r \geq 0$ be the compound interest rate and $U_{0}=x$ be the initial surplus. Then the surplus at time $n$ is

$$
U_{n}=x(1+r)^{n}+\sum_{k=1}^{n} Y_{k}(1+r)^{n-k+1}-\sum_{k=1}^{n} Z_{k}(1+r)^{n-k}, \quad n \geq 1 .
$$


Here $r$ can be dependent on the time $k$ or even random such that $\left\{r_{k}, k \geq 1\right\}$ is an independent sequence and independent of the sequence $\left\{\left(Z_{k}, Y_{k}\right), k \geq 1\right\}$. But for notational simplicity, we assume that $r$ is a constant. We can model the seasonal effects by assigning different distributions to $\left(Z_{k}, Y_{k}\right)$ for different $k$.

Now we are interested in the asymptotic behavior of the probability

$$
\psi_{n}(x)=\mathbb{P}\left(\inf _{1 \leq k \leq 1} U_{k}<0 \mid U_{0}=x\right) .
$$

Let

$$
\Delta_{k}=Z_{k}-Y_{k}(1+r), \quad X_{k}=\Delta_{k}(1+r)^{-k} \quad \text { and } \quad S_{n}=\sum_{k=1}^{n} X_{k} .
$$

We can rewrite the process $U_{n}$ as

$$
U_{n}=(1+r)^{n}\left(x-S_{n}\right) .
$$

Hence,

$$
\psi_{n}(x)=\mathbb{P}\left(\inf _{1 \leq k \leq n} \frac{U_{k}}{(1+r)^{k}}<0 \mid U_{0}=x\right)=\mathbb{P}\left(\max _{1 \leq k \leq n} S_{k}>x\right)
$$

If we assume that each $\Delta_{k}$, therefore $X_{k}$, is long-tailed, then from (3.3) and Theorem 2.1, we obtain

$$
\psi_{n}(x) \sim \mathbb{P}\left(S_{n}>x\right) .
$$

Now we further assume that the sequence $\left\{\left(Z_{k}, Y_{k}\right), k \geq 1\right\}$ is i.i.d. and that $\Delta_{1}$ follows a Pareto law with tail

$$
\mathbb{P}\left(\Delta_{1}>x\right) \sim x^{-\alpha} L(x)
$$

for some $\alpha>0$ and a function $L(x)$, which slowly varies at infinity. Under these conditions we have from (3.3) and Theorem 2.2 that

$$
\begin{aligned}
\psi_{n}(x) & \sim \sum_{k=1}^{n} \mathbb{P}\left(X_{k}>x\right)=\sum_{k=1}^{n} \mathbb{P}\left(\Delta_{1}(1+r)^{-k}>x\right) \\
& \sim \sum_{k=1}^{n}(1+r)^{k \alpha} \mathbb{P}\left(\Delta_{1}>x\right) \\
& \sim \frac{(1+r)^{(n+1) \alpha}-(1+r)^{\alpha}}{(1+r)^{\alpha}-1} x^{-\alpha} L(x),
\end{aligned}
$$

where the coefficient in the last step naturally takes value of $n$ provided that $r=0$.

Example 3.2. In the classical insurance risk models the collective risk process $W(t)$ is often in the form of (1.2). In a recent paper by $\mathrm{Ng}$ et al. (2001), we 
proposed a customer-arrival based model for $W(t)$. Such a $W(t)$ may or may not be infinitely divisible. We assume that the customer arrival process $N(t)$ is a counting process. When the $k$ th $(k \geq 1)$ customer arrives, he/she buys an insurance contract. The insurance company will therefore bear an underlying risk from this policy holder within a fixed term, say $T$. Suppose that the total potential claims due to the $k$ th customer within the term $T$ is $Z_{k}$, and that $\left\{Z_{k}, k \geq 1\right\}$ forms a sequence of i.i.d. non-negative r.v.'s with a finite expectation $\mu$ and independent of the customer-arrival process $N(t)$. The price of each policy is $(1+\delta) \mu$, where the constant $\delta>0$ can be interpreted as the safety loading coefficient. The collective risk process of the company within the period $[0, t]$ in the customer-arrival based model is

$$
W(t)=\sum_{k=1}^{N(t)}\left(Z_{k}-(1+\delta) \mu\right), \quad t \geq 0 .
$$

We write $X_{k}=Z_{k}-(1+\delta) \mu, k \geq 1$, and denote by $F$ the distribution of $X_{1}$. In view of Theorem 2.3, if we assume $F \in L \cap \mathcal{D}$ and that

$$
\mathbb{P}(N(1)>x)=o(\bar{F}(x)),
$$

then for the collective risk process in (3.5) we have

$$
\mathbb{P}\left(\sup _{0 \leq t \leq 1} W(t)>x\right) \sim \mathbb{P}(W(1)>x) \sim \mathbb{E} N(1) \bar{F}(x) .
$$

We note that (3.6) is a very weak condition. It can be satisfied by many commonly used counting processes such as the ordinary renewal process and the compound Poisson process. More generally, (3.6) can be satisfied by the compound renewal process, as demonstrated by the following

Lemma 3.3. Consider the compound renewal process

$$
N(t)=\sum_{k=1}^{\infty} Y_{k} \mathbb{1}_{\left(\sigma_{k} \leq t\right)}, \quad t \geq 0,
$$

where, $\sigma_{k}$ denotes the kth partial sum of an i.i.d. and non-negative sequence $\left\{\theta_{k}\right.$, $k \geq 1\}$, which is independent of another i.i.d., non-negative and integer-valued sequence $\left\{Y_{k}, k \geq 1\right\}$. Let $F \in \mathcal{D}$ be a d.f. with a finite expectation ( $F$ represents the distribution of $X_{1}$ in Example 3.2). Then we have that (3.6) holds if $\mathbb{P}\left(\theta_{1}>0\right)$ $>0$ and $\mathbb{P}\left(Y_{1}>x\right)=o(\bar{F}(x))$.

Proof Let $M(t)$ be an ordinary renewal process driven by the occurrence-times $\left\{\sigma_{k}, k \geq 0\right\}$ with $\sigma_{0}=0$, i.e.,

$$
M(t)=\max \left\{k \geq 0: \sigma_{k} \leq t\right\}, \quad t \geq 0 .
$$


From the assumption $\mathbb{P}\left(\theta_{1}>0\right)>0$ we know that $\mathbb{P}\left(\sigma_{\mathrm{m}_{0}} \leq 1\right)=p_{0}<1$ holds for some $m_{0} \geq 1$. It follows that, for any $n \geq 1$,

$$
\mathbb{P}(M(1) \geq n)=\mathbb{P}\left(\sigma_{n} \leq 1\right) \leq p_{0}^{n / m_{0}}
$$

Hence $M(1)$ has exponential moments. We remark that a short proof of the fact that $M(1)$ has exponential moments can also be found in Smith (1958). Now we start to prove (3.6). By Lemma 3.2 in Tang et al. (2001) we can obtain that, for all $u>0, x>0$ and $n \geq 1$,

$$
\mathbb{P}\left(\sum_{k=1}^{n} Y_{k}>x\right) \leq n \mathbb{P}\left(Y_{1}>x / u\right)+(e d n / x)^{u},
$$

where $d$ is the expectation of $Y_{1}$. Note that $d<\infty$ can be implied by the assumption $\mathbb{P}\left(Y_{1}>x\right)=o(\bar{F}(x))$. We choose $u>0$ in (3.8) sufficiently large such that $x^{-u}=\mathrm{o}(\bar{F}(x))$; for details of the existence of the mentioned $u$, please refer to Theorem 2.2.7 in Bingham et al. (1987), in which we can find a useful representation for the tail probability $\bar{F}(x)$ for $F \in \mathcal{D}$. Thus,

$$
\begin{aligned}
\mathbb{P}(N(1)>x) & =\sum_{n=1}^{\infty} \mathbb{P}\left(\sum_{k=1}^{n} Y_{k}>x\right) \mathbb{P}(M(1)=n) \\
& \leq \sum_{n=1}^{\infty}\left(n \mathbb{P}\left(Y_{1}>x / u\right)+(e d n / x)^{u}\right) \mathbb{P}(M(1)=n) \\
& =\mathbb{E} M(1) \mathbb{P}\left(Y_{1}>x / u\right)+(e d l x)^{u} \mathbb{E} M^{u}(1) \\
& =o(\bar{F}(x)),
\end{aligned}
$$

where in the last step we have used the fact that

$$
\bar{F}(x / u)=\bar{F}(x), \quad \text { for any } u>0
$$

which is implied by the assumption $F \in \mathcal{D}$. This ends the proof.

\section{Proofs of Main Results}

\subsection{Lemmas}

In this section, all the notations are the same as those given at the beginning of Section 2. Before we give the proofs of the main results, we present some important lemmas.

The first lemma below is from Petrov (1975), the two inequalities of which can be regarded as extensions of the classical Lévy's inequality and Kolmogorov's inequality respectively. For $0<q<1$, we denote by $\gamma_{q}(X)$, the quantile 
of order $q$ of a r.v. $X$; that is, the value of $\gamma_{q}(X)$ can be arbitrarily chosen from the set

$$
\Gamma_{q}(X)=\{x: \mathbb{P}(X \leq x) \geq q, \mathbb{P}(X \geq x) \geq 1-q\} .
$$

Lemma 4.1. 1. For any $0<q<1$ and real value of $x$ it holds that

$$
\mathbb{P}\left(\max _{1 \leq k \leq n} S_{k} \geq x\right) \leq \frac{1}{q} \mathbb{P}\left(S_{n} \geq x-\max _{1 \leq k \leq n}\left\{\gamma_{q}\left(S_{k}-S_{n}\right)\right\}\right) .
$$

2. Let $\mathbb{E}\left|X_{k}\right|<\infty$ for $1 \leq k \leq n$. Then, for any $0<q<1$ and real value of $x$ it holds that

$$
\mathbb{P}\left(\max _{1 \leq k \leq n} S_{k} \geq x\right) \leq \frac{1}{q} \mathbb{P}\left(S_{n} \geq x-\frac{\sum_{k=1}^{n} \mathbb{E}\left|X_{k}\right|}{1-q}\right) .
$$

Next, we put forward another lemma, which shows the closure property of class $\mathcal{L}$ under convolution; for some closely related discussions, we refer to Willekens (1986).

Lemma 4.2. Let the d.f. $F_{k}$ belong to class $\iota, k \geq 1$. Then we have, for any $n \in \mathcal{N}$

$$
V=F_{1} * F_{2} \cdots * F_{n} \in \mathcal{L} \text {. }
$$

Proof. It suffices to show the assertion for the case $n=2$. From the definition of (2.3), this amounts to showing that $V=F_{1} * F_{2}$ satisfies

$$
\bar{V}(x+1) \sim \bar{V}(x) .
$$

For arbitrarily fixed $0<M<x$, we write

$$
\bar{V}(x)=\int_{-\infty}^{\infty} \bar{F}_{1}(x-t) F_{2}(d t)=I_{1}+I_{2},
$$

where $I_{1}$ and $I_{2}$ are the integrals of $\bar{F}_{1}(x-t)$ with respect to $F_{2}(d t)$ over $(-\infty$, $x-M]$ and $(x-M, \infty)$ respectively. By the same method we also divide the tail $\bar{K}(x+1)$ into two parts as $J_{1}+J_{2}$, where $J_{i}$ is the integral of $\bar{F}_{1}(x+1-t)$ with respect to $F_{2}(d t)$ over the same interval as that of $I_{i}, i=1,2$. First we start to compare $I_{1}$ with $J_{1}$. Clearly, we have

$$
\inf _{t \geq M} \frac{\overline{F_{1}}(t)}{\bar{F}_{1}(t+1)} \leq \frac{I_{1}}{J_{1}}=\frac{\int_{-\infty}^{x-M} \overline{F_{1}}(x-t) F_{2}(d t)}{\int_{-\infty}^{x-M} \bar{F}_{1}(x+1-t) F_{2}(d t)} \leq \sup _{t \geq M} \frac{\overline{F_{1}}(t)}{\overline{F_{1}}(t+1)}
$$

Therefore, by the definition of (2.3), we see that

$$
\lim _{M \rightarrow \infty} \liminf _{x \rightarrow \infty} \frac{I_{1}}{J_{1}}=\lim _{M \rightarrow \infty} \liminf _{x \rightarrow \infty} \frac{I_{1}}{J_{1}}=1 .
$$


Now we turn to compare $I_{2}$ with $J_{2}$. Again by the fact that $F_{2} \in \mathcal{L}$, we can see that, for any fixed $M>0$,

$$
\begin{aligned}
I_{2} & =\int_{x-M}^{x} \overline{F_{1}}(x-t) F_{2}(d t)+\int_{x}^{\infty}(x-t) F_{2}(d t) \\
& =o\left(\overline{F_{2}}(x)\right)+\overline{F_{1}}(0) \overline{F_{2}}(x)+\int_{-\infty}^{0} \overline{F_{2}}(x-t) F_{1}(d t) \\
& \sim \bar{F}_{2}(x) .
\end{aligned}
$$

By the same approach we can also derive that $J_{2} \sim \overline{F_{2}}(x)$. Hence, for arbitrarily fixed $0<M<x$,

$$
I_{2} \sim J_{2}
$$

We conclude from (4.4), (4.5) and (4.6) that (4.3) holds.

This ends the proof.

Finally we state an inequality for the tail probabilities of sums of i.i.d. r.v.'s with dominatedly varying tails.

Lemma 4.3. Let $G$ be a d.f. supported on $[0, \infty)$ and belonging to $\mathcal{D}$ with a finite expectation $\mu$. Then, for any $\gamma>\mu$ there exists a constant $C=C(\gamma)>0$ such that

$$
\overline{G^{* n}}(x) \leq C n \bar{G}(x)
$$

for all $n \geq 1$ and all $x \geq \gamma n$.

Proof. The proof of a similar but more precise result can be found in $\mathrm{Ng}$ et al. (2001). Here we provide a few key steps of the proof.

Let $\left\{X_{n}, n \geq 1\right\}$ be a sequence of i.i.d. r.v.'s with a common d.f. $G$ above. We write

$$
\widetilde{X_{n}}=X_{n} I_{\left(X_{n \leq l x}\right)} \text { and } \widetilde{S_{n}}=\sum_{i=1}^{n} \widetilde{X_{i}} \text { for } n \geq 1 \text {, }
$$

where $0<l<1$ is a constant. Obviously,

$$
\begin{aligned}
\overline{G^{* n}}(x) & \leq P\left(\max _{l \leq i \leq n} X_{i}>l x\right)+P\left(\max _{l \leq i \leq n} X_{i} \leq l x, S_{n}>x\right) \\
& \leq n \bar{G}(l x)+P\left(\widetilde{S_{n}}>x\right) .
\end{aligned}
$$

The upper bound, say $C_{1}(l) n \bar{G}(x)$, for the first term in (4.8) can be obtained by the fact that $G \in \mathcal{D}$ whenever the constant $l$ is fixed. 
Let $a=\max \{-\log (n \bar{G}(x))$. Clearly, $a$ tends to $\infty$ uniformly for $x \geq \gamma n$ as $n \rightarrow \infty$. For any $\mathrm{h}>0$, we have

$$
\begin{aligned}
\frac{P\left(\widetilde{S_{n}}>x\right)}{n \bar{G}(x)} & \leq \frac{e^{-h x} E e^{h S_{n}}}{n \bar{G}(x)} \leq e^{-h x+a}\left\{\int_{0}^{l x}\left(e^{h t}-1\right) G(d t)+1\right\}^{n} \\
& \leq \exp \left\{n\left(\int_{0}^{l x l a}+\int_{l x d a}^{l x}\right)\left(e^{h t}-1\right) G(d t)-h x+a\right\} \\
& =\exp \left\{n\left(K_{1}+K_{2}\right)-h x+a\right\} .
\end{aligned}
$$

We can obtain the required upper bound for $K_{1}$ by using $e^{u}-1 \leq u e^{u}$ for any real number $u$. In addition, Proposition 2.2.1 in Bingham et al. (1987) stated that, for $G \in \mathcal{D}$, there exist positive $x_{0}, \rho$ and $B$ such that

$$
\bar{G}(u x) / \bar{G}(x) \leq B u^{-\rho}
$$

uniformly for all $x \geq x_{0} / l$ and all $0<u<1$. This result, with $u$ in (4.10) replaced by $l / a$, can be used to derive the required upper bound for $K_{2}$.

Substituting the upper bounds for $K_{1}$ and $K_{2}$ into (4.9) and choosing the constants $h$ and $l$ carefully, we can prove that the right-hand side of (4.9) is bounded by a sufficiently large positive constant, say $C_{2}(l, \gamma)>0$. Therefore the second term in (4.8) is bounded by $C_{2}(l, \gamma) n \bar{G}(x)$ uniformly for $n \geq 1$ and $x \geq \gamma n$. From this (4.7) can be finally proven.

\subsection{Proofs of Theorems}

\section{Proof of Theorem 2.1.}

In order to prove (2.5) we need to show that

$$
\mathbb{P}\left(\max _{1 \leq k \leq n} S_{k}>x\right) \leq \mathbb{P}\left(S_{n}>x\right) \text { and } \mathbb{P}\left(\max _{1 \leq k \leq n} S_{k}>x\right) \geq \mathbb{P}\left(S_{n}>x\right) \text {. }
$$

The last relation in (4.11) is obvious. By Lemma 4.2 we know the distribution of $S_{n}$ belongs to class $\mathcal{L}$. Then by (4.1), we have, for any $0<q<1$,

$$
\mathbb{P}\left(\max _{1 \leq k \leq n} S_{k}>x\right) \lesssim \frac{1}{q} \mathbb{P}\left(S_{n} \geq x-\max _{1 \leq k \leq n}\left\{\gamma_{q}\left(S_{k}-S_{n}\right)\right\}\right) \sim \frac{1}{q} \mathbb{P}\left(S_{n} \geq x\right) .
$$

So letting $q \nearrow 1$ in the above yields the first relation in (4.11).

\section{Proof of Theorem 2.2.}

Noting the fact $\mathcal{L} \subset \mathcal{L}$, we see that $\mathbb{P}\left(\max _{1 \leq k \leq n} S_{k}>x\right) \sim \mathbb{P}\left(S_{n}>x\right)$ is the immediate consequence of Theorem 2.1. The result $\mathbb{P}\left(S_{n}>x\right) \sim C(n) \bar{F}(x)$ is a particular case $(y=0)$ of the Proposition in Sgibnev (1988). In addition, one easily obtains $\mathbb{P}\left(\max _{1 \leq k \leq n} X_{k}>x\right) \sim C(n) \bar{F}(x)$ by induction. This ends the proof of Theorem 2.2. 


\section{Proof of Theorem 2.3.}

In order to prove (2.7), we consider it as a conjunction of two asymptotic relationships,

$$
\mathbb{P}\left(\max _{1 \leq k \leq \tau} S_{k}>x\right) \sim \mathbb{E} \tau \bar{F}(x) \text { and } P\left(S_{\tau}>x\right) \sim \mathbb{E} \tau \bar{F}(x) .
$$

We write $X_{k}^{+}=\max \left\{X_{k}, 0\right\}, \mu^{+}=\mathbb{E} X_{k}^{+}$, and let the d.f. $G$ in Lemma 4.3 be the distribution of the r.v. $X_{k}^{+}$. For a fixed $0<q<1$, we choose a sufficiently large constant $\gamma$ such that

$$
\gamma>\mu^{+}+\frac{\mathbb{E}\left|X_{1}\right|}{1-q} .
$$

Now we derive

$$
\begin{aligned}
\mathbb{P}\left(\max _{1 \leq k \leq \tau} S_{k}>x\right) & =\sum_{n=1}^{\infty} \mathbb{P}\left(\max _{1 \leq k \leq n} S_{k}>x\right) \mathbb{P}(\tau=n) \\
& =\left(\sum_{1 \leq n<x / \gamma}+\sum_{n \geq x / \gamma}\right) \mathbb{P}\left(\max _{1 \leq k \leq n} S_{k}>x\right) \mathbb{P}(\tau=n) \\
& =L_{1}+L_{2} .
\end{aligned}
$$

For $1 \leq n<x / \gamma$, we obtain, successively by (4.2) and (4.7),

$$
\begin{aligned}
\mathbb{P}\left(\max _{1 \leq k \leq n} S_{k}>x\right) & \leq \frac{1}{q} \mathbb{P}\left(\sum_{k=1}^{n} X_{k}^{+} \geq x-\frac{n \mathbb{E}\left|X_{1}\right|}{1-q}\right) \\
& \leq \frac{C n}{q} \mathbb{P}\left(X_{1}^{+} \geq x-\frac{n \mathbb{E}\left|X_{1}\right|}{1-q}\right) \\
& \leq \frac{C n}{q} \mathbb{P}\left(X_{1}^{+} \geq x\left(1-\frac{\mathbb{E}\left|X_{1}\right|}{\gamma(1-q)}\right)\right) \\
& \simeq n \mathbb{P}\left(X_{1}^{+} \geq x\right) \\
& =n \bar{F}(x),
\end{aligned}
$$

where, in (4.14) we have used (3.9). So by the dominated convergence theorem and Theorem 2.2 we conclude

$$
L_{1} \sim \sum_{1 \leq n<x d y} n \bar{F}(x) \mathbb{P}(\tau=n) \sim \mathbb{E} \tau \bar{F}(x)
$$

Additionally, by the condition that $\mathbb{P}(\tau>x)=o(\bar{F}(x))$, we have

$$
L_{2} \leq \mathbb{P}(\tau \geq x / \gamma)=o(\bar{F}(x / \gamma))=o(\bar{F}(x))
$$


Substituting (4.15) and (4.16) into (4.13), we obtain the first asymptotic relationship in (4.12). Finally, we have

$$
\begin{aligned}
\mathbb{P}\left(\max _{1 \leq k \leq \tau} S_{k}>x\right) & \geqslant \mathbb{P}\left(S_{\tau}>x\right) \\
& \gtrless \sum_{1 \leq n<x / \gamma} \mathbb{P}\left(S_{n}>x\right) \mathbb{P}(\tau=n) \\
& \sim \sum_{1 \leq n<x / y} n \bar{F}(x) \mathbb{P}(\tau=n) \\
& =\mathbb{E} \tau \bar{F}(x) .
\end{aligned}
$$

This, coupled with the first asymptotic relationship in (4.12), implies the second in (4.12). The proof of Theorem 2.3 is completed.

\section{ACKNOWLEDGMENTS}

The authors would like to thank the referees for helpful suggestions and comments. This research was conducted while the second author was visiting the Department of Statistics and Actuarial Science, the University of Hong Kong during the period from May to November of year 2001. The second author would like to thank the department for the kind hospitality. This work was supported by Research Grants Council of HKSAR (Project No: HKU 7139/01H).

\section{REFERENCES}

BERMAN, S.M. (1986) The supremum of a process with stationary independent and symmetric increments. Stochastic Process. Appl., 23(2): 281-290.

Bingham, N.H., Goldie, C.M. and Teugels, J.L. (1987) Regular Variation. Cambridge University Press, Cambridge.

Braverman, M. (1999) Remarks on suprema of Lévy processes with light tails. Statist. Probab. Lett., 43(1): 41-48.

Braverman, M. (2000) Suprema of compound Poisson processes with light tails. Stochastic Process. Appl., 90(1), 145-156.

Braverman, M. and SAMORODNITSKy, G. (1995) Functionals of infinitely divisible stochastic processes with exponential tails. Stochastic Process. Appl., 56(2): 207-231.

Chover, J., NeY, P. and WaInger, S. (1973) Functions of probability measures. J. Analyse Math., 26: 255-302.

Cline, D.B.H. (1986) Convolution tails, product tails and domains of attraction. Probab. Theory Relat. Fields., 72(4), 529-557.

Cline, D.B.H. (1987) Convolutions of distributions with exponential and subexponential tails. J. Austral. Math. Soc. Ser. A, 43: 347-365.

Embrechts, P., Goldie, C.M. and Veraverbeke, N. (1979) Subexponentiality and infinite divisibility. Z. Wahrscheinlichkeitstheorie verw. Gebiete, 49: 335-347.

Embrechts, P. and Goldie, C.M. (1982) On convolution tails. Stoch. Proc. Appl, 13: 263-278.

EMBREChTs, P., KLÜPPELBERG, C. and Miкosch, T. (1997) Modelling Extremal Events for Insurance and Finance. Springer, Berlin.

GoLDIE, C.M. and KuÜPPELBERG, C. (1998) Subexponential distributions. A practical Guide to HeavyTails: Statistical Techniques and Applications. Eds. Adler, R.J., Feldman, R.E., Taqqu, M.S. Birkhäuser. 
NG, K.W., TANG, Q.H., YAN, J.A. and YANG, H. (2001) Large deviation results for collective insurance risk processes with heavy-tailed claims and applications. Submitted.

Petrov, V.V. (1975) A generalization of an inequality of Lévy. Theory Prob. Appl., 20(1): 141145.

SGlBNEV, M.S. (1988) Banach algebras of measures of class S( $\gamma$ ). Siberian Math. J., 29: 647-655.

SGIBNEv, M.S. (1996) On the distribution of the maxima of partial sums. Statistics \& Probability Letters, 28: 235-238.

SMITH, W.L. (1958) Renewal theory and its ramifications. J. Roy. Statist. Soc. Ser. B, 20: 243302.

TANG, Q.H., SU, C., JIANG, T. and ZhanG, J.S. (2001) Large deviations for heavy-tailed random sums in compound renewal model. Stat. Prob. Letters, 52(1): 91-100.

Willekens, E. (1986) Subexponentiality on the real line. Technical Report, K.U. Leuven.

WILLEKENS, E. (1987) On the supremum of an infinitely divisible process. Stochastic Process. Appl., 26(1): 173-175.

YANG, H. (1999) Non-exponential bounds for ruin probability with interest effect included. Scandinavian Actuarial Journal, 66-79.

K.W. NG

Department of Statistics and Actuarial Science

University of Hong Kong

Pokfulam Road, Hong Kong

E-mail: kaing@hku.hk

Q.H. TANG

University of Amsterdam

Department of Quantitative Economics

Roetersstraat 11,1018 WB Amsterdam, The Netherlands

E-mail:tangqihe@263.net

H. YANG

Department of Statistics and Actuarial Science

University of Hong Kong

Pokfulam Road, Hong Kong

E-mail: hlyang@hku.hk 